\title{
Periprostatic Haematoma After Transrectal Ultrasound Guided Biopsy - The Importance of Multidisciplinary Team Approach: Case Report
}

\author{
Michal Sut ${ }^{\mathrm{a}, \mathrm{c}}$, Asheesh Kaul ${ }^{\mathrm{a}}$, Christopher Jacobs ${ }^{\mathrm{a}}$, Ramsay Bowden ${ }^{\mathrm{a}}$, \\ Muhammad Shaikh ${ }^{\mathrm{b}}$, Aasem Chaudry ${ }^{\mathrm{a}}$
}

\begin{abstract}
We present a case of a 64-year-old man who was diagnosed with Gleason $3+4=7$ adenocarcinoma of the prostate following transrectal ultrasound guided prostatic biopsy for elevated PSA. Staging Magnetic Resonance scan revealed a well defined lesion between right prostatic lobe and the rectum. Signal changes were atypical for haematoma in follow up scans. We discuss diagnostic process and the impact of multidisciplinary approach.
\end{abstract}

Keywords: PSA; Prostatic biopsy; Hematoma

\section{Introduction}

Extraprostatic haematomas following transrectal ultrasound guided biopsies are rare and may therefore represent a significant diagnostic challenge. Involvement of the Multidisciplinary Team (MDT) including dedicated genitourinary Radiologist in the decision making process is of a paramount importance.

\section{Case Report}

We present a case of a 64-year-old patient referred to Urology Department in Bedford Hospital with an elevated PSA of $5.5 \mathrm{ng} / \mathrm{mL}$. Following rectal examination revealing enlarged and smooth prostate gland he was counseled and referred for transrectal ultrasound guided prostatic biopsy. Histology re-

\footnotetext{
Manuscript accepted for publication October 4, 2012

aUrology Department, Bedford Hospital NHS Trust, Kempston Road, Bedford MK42 9DJ, UK

${ }^{\mathrm{b}}$ Radiology Department, Bedford Hospital NHS Trust, Kempston Road, Bedford MK42 9DJ, UK

${ }^{\mathrm{c} C}$ Corresponding author: Michal Sut, 19 Palgrave Road, Bedford MK42 9DH, UK. Email: michalsut@yahoo.co.uk
}

doi: http://dx.doi.org/10.4021/wjnu52w port revealed benign left sided biopsy cores and a Gleason 3 $+4=7$ adenocarcinoma in 4 out of 6 cores involving $30 \%$ of the tissue from the right prostatic lobe. On the basis of those findings local staging was advocated by Multidisciplinary Team (MDT).

Magnetic Resonance (MR) scan without endorectal coil was performed 16 days following biopsy and revealed neither extraprostatic extension, seminal vesicle invasion or significant intrapelvic lymphadenopathy. A well defined rounded lesion measuring $36 \times 28 \mathrm{~mm}$ was noted posteriorly between the right prostatic lobe and the rectum causing smooth indentation to both organs without any local infiltration (Fig.1, 2). The lesion showed slightly heterogenous low signal in the centre and thick peripheral rim of the high signal on $\mathrm{T} 1 \mathrm{~W}$, T2W and T2FATSAT sequences. No enhancement was noted after administration of intravenous gadolinium. Follow up MR scans 45 and 82 days after biopsy revealed size reduction to $22 \times 16 \mathrm{~mm}$ and $16 \times 10 \mathrm{~mm}$ respectively (Fig. 3). The signal characteristics of the lesion showed no significant change during this period. The appearances were not entirely consistent with those of a chronic pelvic haematoma [1].

Findings were re-discussed at the MDT meeting with the presence of Oncology, Pathology, Radiology, and Urology

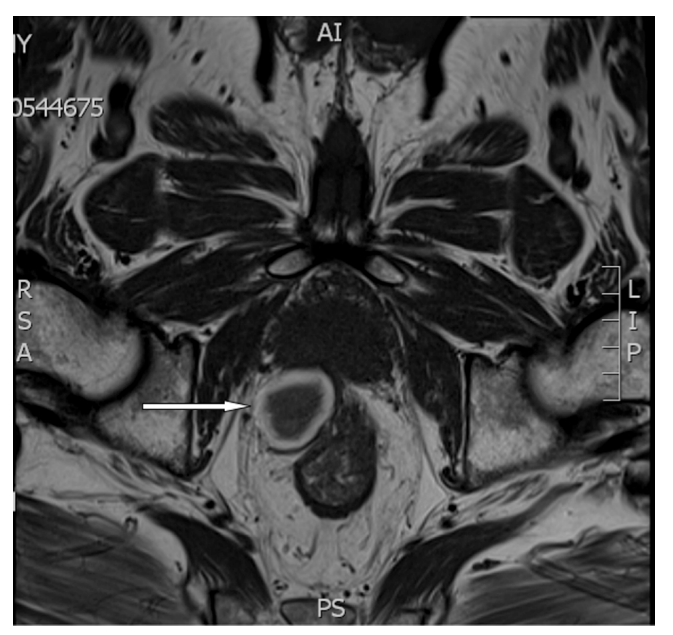

Figure 1. (MR) Axial T1W sequence shows low central signal and peripheral rim of high signal in the lesion. 


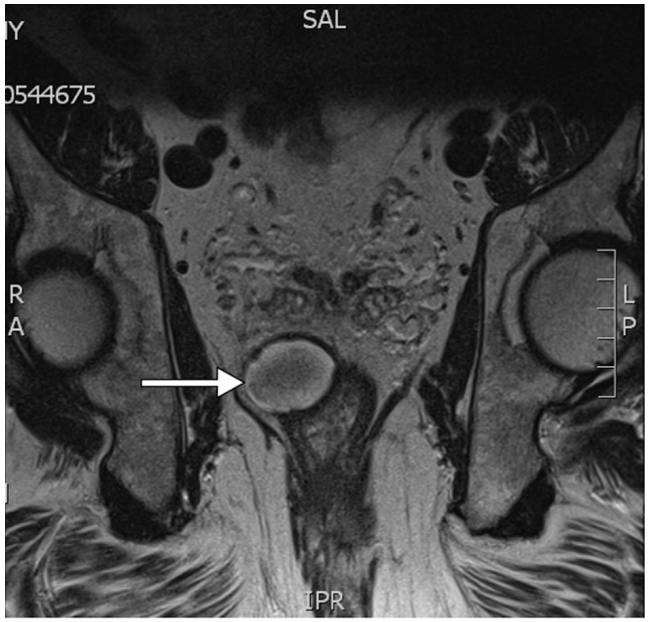

Figure 2. (MR) Coronal T2W showing smooth indentation to rectum. The lesion is of low signal centrally with a rim of high signal.

Consultants and on the basis of benign appearance, decreasing size and despite the lack of typical signal changes it was concluded to be a benign finding, most likely biopsy related resolving haematoma. Patient was offered radical treatment in a form of radiotherapy.

\section{Discussion}

Despite technological advances in the field of medical imaging and extensive research on PSA and other prostate cancer markers ultrasound guided biopsy remains the gold standard in establishing prostate cancer diagnosis. Whether it is performed transrectally or transperineally it carries quite significant risks related to bleeding, infection and urinary retention. In UK practice positive biopsy results are discussed at MDT meetings where the risk stratification and treatment intentions are taken under consideration in accordance with local policy and national guidance [2] to determine the diagnostic imaging protocol.

MR scan is used for staging of suspected local disease as it provides superior assessment of extracapsular extension and seminal vesicle invasion comparing to Positron Emission Computed Tomography and Transrectal Ultrasound [3] especially when endorectal coil is used and images are reported by genitourinary Radiologist [4].

Difficult interpretation of MR images due to intraprostatic haemorrhage following prostatic biopsies has been de-

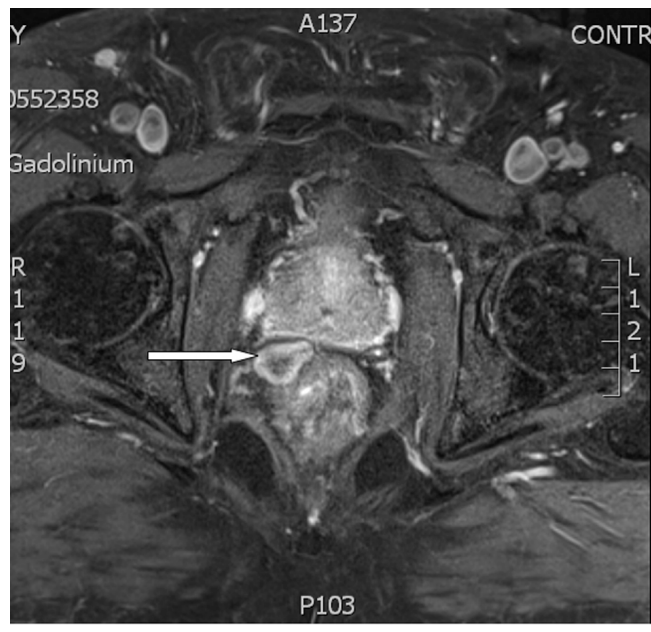

Figure 3. (MR) Axial T1FS sequence at 82 days showing reduction in size.

scribed [5]. Conversely, extraprostatic haematomas are rare and could therefore represent a diagnostic challenge. We believe that multidisciplinary approach and especially involvement of a dedicated genitourinary Radiologist is of a paramount importance and as in presented case it facilitates consensus, minimizes the extent of diagnostic route and expedites therapeutic decision.

\section{References}

1. Yamashita Y, Hatanaka Y, Torashima M, Takahashi M. Magnetic resonance characteristics of intrapelvic haematomas. Br J Radiol. 1995;68(813):979-985.

2. NICE Prostate Cancer Guidelines.

3. Rinnab L, Blumstein NM, Mottaghy FM, Hautmann RE, Kufer R, Hohl K, Reske SN. 11C-choline positronemission tomography/computed tomography and transrectal ultrasonography for staging localized prostate cancer. BJU Int. 2007;99(6):1421-1426.

4. Mullerad M, Hricak H, Wang L, Chen HN, Kattan MW, Scardino PT. Prostate cancer: detection of extracapsular extension by genitourinary and general body radiologists at MR imaging. Radiology. 2004;232(1):140146.

5. White S, Hricak H, Forstner R, Kurhanewicz J, Vigneron DB, Zaloudek CJ, Weiss JM, et al. Prostate cancer: effect of postbiopsy hemorrhage on interpretation of MR images. Radiology. 1995;195(2):385-390. 